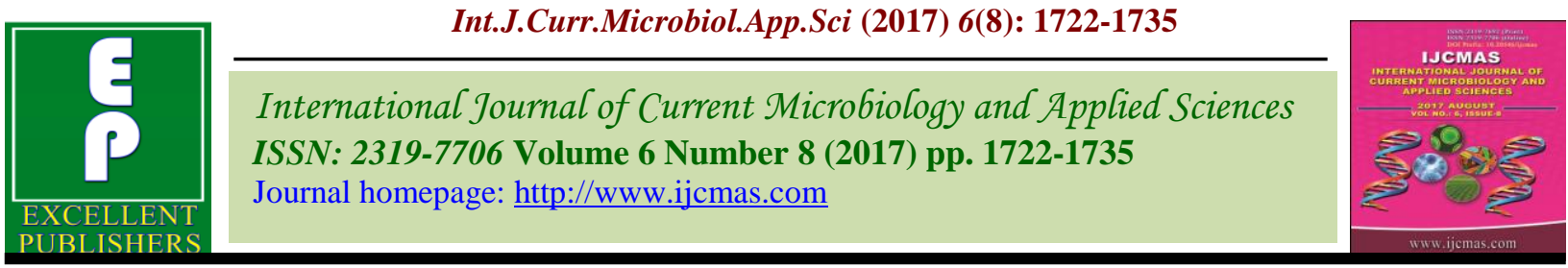

Original Research Article https://doi.org/10.20546/ijcmas.2017.608.206

\title{
Genetic Studies for Yield and Yield Components in Inter-Specific Hybrids (G. hirsutum × G. barbadense) of Cotton Over Environments
}

\author{
B. Balakrishna*, Chenga Reddy, K.V. Siva Reddy and M. Lal Ahamed \\ Regional Agricultural Research Station and All India Co-ordinated Cotton Improvement Project \\ Sub Center, Cotton section, Lam farm, Guntur, Andhra Pradesh- 522 034, India \\ *Corresponding author
}

\begin{tabular}{|l|}
\hline Ke y w o r d s \\
Cotton, Inter- \\
specific hybrids, \\
Combining \\
ability, Heterosis, \\
Gene action and \\
Over \\
environments.
\end{tabular}

\section{Introduction}

The knowledge of genetic structure and mode of inheritance of quantitative and qualitative traits helps the breeders to employ suitable breeding method for improvement of these traits. Combining ability analysis is an important tool for selection of the desirable parents together with the information regarding nature and magnitude of gene action controlling quantitative and qualitative traits. The information on gene action and combining ability over locations (pooled) helps in identifying the best combiners which may be hybridized to produce superior stable heterotic cross combinations or to produce transgressive or recombinant segregants in $\mathrm{F}_{2}$ and subsequent generations. The entire genetic variability observed in the analysis for each trait was partitioned into its components i.e., general and specific combining ability (Sprague and Tatum, 1942). General combining ability $(\mathrm{gca})$ is used to designate the average performance of a line in hybrid 
combinations while specific combining ability (sca) define those cases in which certain combinations do relatively better or worse than expected on the basis of the average performance of the lines involved. The relative importance of general and specific combining ability can be assessed by estimating the components of variance and expressed in the ratio of $\sigma^{2} g c a / \sigma^{2} s c a$ (Singh and Chaudhary, 1977). The closer the ratio to unity, greater is the magnitude of additive gene action and vice versa.

The purpose of this study was to determine the $g c a$ and $s c a$ effects for seed cotton yield plant $^{-1}$, yield contributing and fibre quality traits so as to choose appropriate parents and hybrids for the genetic enhancement. Study on the magnitude of heterosis in $F_{1} \mathrm{~s}$ helps to assess the ability of the test genotypes to transmit the desirable characters to the $F_{1} S$ upon hybridization. This facilitates in identification of genotypes that exhibit high amount of exploitable heterosis.

\section{Materials and Methods}

The present investigation was carried over three locations i.e., Regional Agricultural Research Station (RARS), Lam Farm, Agricultural Research Station (ARS), Jangamaheswarapuram and Agricultural College Farm, Bapatla during kharif 2014-15. During off-season 2013-14, 12 hirsutum lines viz., NDLH 1938, H 1442, ADB 542, ADB 532, WGCV 48, MR 786, TSH 0250, BS 37, SCS 793, MCU 5, L 1058, L 762 and six barbadense testers viz., GSB 40, DB 16, DB 11, GSB 41, TCB 37 and SUVIN were mated in line $\times$ tester fashion and obtained 72 inter-specific cross combinations. The hybrids along with their parents and two checks (DCH 32 and Mahalakshmi) i.e., 96 genotypes were evaluated in a Randomized Complete Block design with three replications. Each plot consisted of two rows of $10 \mathrm{~m}$ length spaced at $1.20 \mathrm{~m}$ apart with plant to plant distance of
$60 \mathrm{~cm}$. All the recommended agronomical practices and plant protection measures were followed as and when required to raise a good crop of cotton. The data was recorded from five randomly selected from each cross combination for yield and yield components. The data was subjected to statistical analysis for calculating combining ability variances and effects using line $x$ tester method suggested by Kempthorne (1957) and detailed by Singh and Chaudhary (1985). Standard heterosis was calculated over both the checks by adopting methodology suggested by Meredith and Bridge (1972).

\section{Results and Discussion}

\section{Line $\times$ tester pooled analysis for combining ability}

The pooled analysis of variance for combining ability over three locations recorded significant differences for all the characters studied (Table 1). The differences among the parents, parents' vs. crosses and crosses were observed to be significant for all the characters studied. Partitioning of crosses into lines, testers and line $\times$ tester revealed that the variance due to lines was significant for all the characters whereas, for testers all the characters were found to be significant except for days to $50 \%$ flowering, number of monopodia plant $^{-1}$, number of bolls plant ${ }^{-1}$ and seed index.. The interaction due to lines $x$ testers were significant for all the traits evaluated. Interaction effect of locations $x$ parents' $v s$. crosses, locations $\times$ crosses were significant for all the characters. Further, locations $\times$ line effect interaction showed significant differences for days to $50 \%$ flowering, plant height, number of monopodia plant $^{-1}$, number of sympodia plant ${ }^{-1}$, number of bolls plant ${ }^{-1}$ and seed cotton yield plant ${ }^{-1}$. While, locations $\times$ tester effect interaction exhibited non-significant for all the characters except for number of monopodia plant ${ }^{-1}$. Interaction effects of locations $\times$ line $\times$ testers 
were significant for all the characters studied. General combining ability effects of parents and specific combining ability effects of crosses over locations (pooled) were estimated and presented in tables 2 and 3.

\section{Days to 50\% flowering}

General combining ability effects for days to $50 \%$ flowering in pooled analysis varied from -2.88 (L 762) to 2.12 (BS 37) in lines and from -1.09 (DB 16) to 1.15 (TCB 37) in testers. The lines, L 762 (-2.88), H 1442 (1.90) and NDLH $1938(-0.64)$ and the testers DB $16\left(-1.09^{*}\right)$ and GSB $41(-0.45)$ recorded significant $g c a$ effects in desired direction for this trait and these parents were good general combiners with favourable genes for earliness over locations. In pooled analysis, estimates of specific combining ability effects for this trait ranged from $-5.72(\mathrm{H} 1442 \times \mathrm{GSB} 41)$ to 5.70 (L $1058 \times$ DB 16). Out of 72 cross combinations evaluated, seventeen hybrids recorded significant negative sca effects. When compared over their per se performance and sca effects the crosses, $\mathrm{H}$ $1442 \times$ GSB $41($ High $\times$ High $)$, TSH $0250 \times$ GSB 40 (Low $\times$ Low) and NDLH $1938 \times$ DB 11 (High $\times$ Low) were identified as best specific cross combinations for this trait.

Days to $50 \%$ flowering in pooled analysis recorded significant negative standard heterosis over both the checks was recorded by H $1442 \times$ GSB 41(55.67) and L $762 \times$ DB 16 (58.11) and superiority of these hybrids was further confirmed by their per se performance and high sca effects. These results are in agreement with the reports of Amalabalu et al., (2012) for heterosis over standard check.

\section{Plant height (cm)}

The gca effects ranged from -8.56 (MCU 5) to 9.32 (NDLH 1938) in lines and from -6.12
(SUVIN) to 5.96 (GSB 40) in testers for plant height across the locations. Significant positive gca effects desirable for this trait was recorded by the lines, NDLH 1938 (9.32**), MR 786 (7.35) and ADB 542 (3.32) and the tester GSB 40 (5.96). These parents can be utilized in basic breeding programmes for improvement of this trait. The estimates of sca effects for plant height varied from -20.14 $($ NDLH $1938 \times$ DB 11) to $19.74($ ADB $532 \times$ DB 16).

Significant positive sca effects in desirable direction was exhibited by ten cross combinations and the top five cross combinations were, ADB $532 \times$ DB 16 (19.74), H $1442 \times$ DB 11 (16.57), TSH 0250 $\times$ GSB 40 (10.79), L $1058 \times$ SUVIN (10.37) and NDLH $1938 \times$ TCB 37 (9.36). The superior cross combinations identified based on their per se performance and sca effects were, H $1442 \times$ DB 11 (Low $\times$ Low), TSH $0250 \times$ GSB 40 (Low $\times$ High) and NDLH $1938 \times$ TCB 37 (High $\times$ Low $)$. The crosses between two low combiners producing crosses with significant $s c a$ effect indicating the presence of non-additive inter-allelic interaction.

Standard heterosis over DCH 32 and Mahalakshmi ranged from -6.56 (ADB $532 \times$ TCB 37) to $21.36(\mathrm{NDLH} 1938 \times$ GSB 40) and from -16.31 (ADB $532 \times$ TCB 37) to 8.70 (NDLH $1938 \times$ GSB 40), respectively. Forty two hybrids over DCH 32 and seventeen hybrids over Mahalakshmi showed significant positive heterosis. Based on overall performance (per se, sca effects and standard heterosis) the top three specific combiners identified for this trait were, NDLH $1938 \times$ GSB 40 (194.54), NDLH $1938 \times$ GSB 41(190.59) and H $1442 \times$ DB 11(189.85). These results are in agreement with the reports of Amalabalu et al., (2012), Kumar et al., (2013) and Nirania et al., (2014) for heterosis over standard check. 
Number of monopodia plant ${ }^{-1}$

A range of -0.31 (NDLH 1938) to 0.51 (BS 37) gca effects was observed for lines. Whereas, testers recorded a range from -0.09 (DB 16) to 0.07 (GSB 40 and SUVIN). Among the lines, BS 37 (0.51), WGCV 48 (0.36), ADB $532(0.18)$, ADB $542(0.10)$ and L 1058 (0.07) while, in testers GSB 40 (0.07), SUVIN (0.07) and GSB 41 (0.04) showed significant positive gca effects over locations. These parents may serve as useful source for improvement of this trait in hybridization programmes. Lowest and highest $s c a$ effects recorded in pooled analysis were $-0.80(\mathrm{MCU}$ $5 \times$ GSB 41) and $0.74($ TSH $0250 \times$ GSB 40). The variance due to $g c a(-0.005)$ was very low and negative than variance due to sca (0.041) and both were non-significant. The crosses, ADB $532 \times$ GSB 41 (High $\times$ High), TSH $0250 \times$ GSB 40 (Low $\times$ High) and MCU $5 \times$ GSB 40 (Low $\times$ High) were identified as best specific combiners as per their per se performance and $s c a$ effects.

Standard heterosis over DCH 32 and Mahalakshmi ranged from -15.19 (TSH $0250 \times$ TCB 37) to $87.94($ ADB $532 \times$ GSB 41) and $-47.26(\mathrm{TSH} 0250 \times \mathrm{TCB} 37)$ to 16.86 (ADB $532 \times$ GSB 41), respectively. Sixty two and eight hybrids showed significant positive heterosis over DCH 32 and Mahalakshmi, respectively. The best heterotic hybrids identified based on per se and sca effects were, ADB $532 \times$ GSB 41(3.20) followed by BS $37 \times$ GSB 40 (3.18) and WGCV $48 \times$ TCB 37 (3.03). These results are in agreement with the reports of Nirania et al., (2014) and Tuteja (2014) for heterosis over standard check.

\section{Number of sympodia plant ${ }^{-1}$}

In pooled analysis $g c a$ effects for number of sympodia plant ${ }^{-1}$ varied from -1.91 (MCU 5) to 1.72 (MR 786) in lines and from -1.24
(TCB 37) to 0.95 (DB 16) in testers. The lines, MR 786 (1.72), SCS 793 (1.06), H 1442 (0.91) and NDLH 1938 (0.40) and the testers DB 16 (0.95), GSB 41 (0.69) and GSB $40\left(0.33^{*}\right)$ exhibited significant and positive gca effects for this trait. Sca effects for this trait ranged from -2.91 (WGCV 48 $\times$ GSB 41) to $5.60($ MR $786 \times$ GSB 41).

The best specific cross combinations identified based on their per se performance and sca effects were MR $786 \times$ GSB 41 $($ High $\times$ High), ADB $542 \times$ DB 16 (Low $\times$ High) and H $1442 \times$ DB $16($ High $\times$ High $)$.

Heterosis over standard checks DCH 32 and Mahalakshmi ranged from -12.52 (MCU $5 \times$ TCB 37) to 36.96 (MR $786 \times$ GSB 41) and $21.46($ MCU $5 \times$ TCB 37) to 22.97 (MR 786 $\times$ GSB 41), respectively. Among seventy two hybrids studied, twenty one and four hybrids exhibited significant positive values over DCH 32 and Mahalakshmi, respectively. Based on per se performance, sca effects and standard heterosis the hybrids viz., MR $786 \times$ GSB 41(31.43), ADB $542 \times$ DB $16(28.02)$ and H $1442 \times$ DB 16 (27.29) were found to be superior. Nirania et al., (2014) and Tuteja (2014) for heterosis over standard check.

\section{Number of bolls plant ${ }^{-1}$}

Among the lines $\mathrm{gca}$ effects ranged from 14.61 (MCU 5) to 6.54 (H 1442) and among the testers, gca effects varied from -2.95 (TCB 37) to GSB 41 (2.71). The lines, H 1442 (6.54), NDLH 1938 (6.43), L 762 (4.41), SCS 793 (3.68), BS 37 (2.68), MR 786 (1.60) and ADB 542 (1.16) and the testers GSB 41 (2.71), DB 16 (2.46) and DB 11 (1.42) recorded significant positive gca effects in pooled analysis. The range of $s c a$ effects was from $-13.96($ TSH $0250 \times$ TCB 37) to $14.26(\mathrm{BS} 37 \times \mathrm{SUVIN})$. The crosses, BS $37 \times$ SUVIN $($ High $\times$ Low $)$, BS $37 \times$ GSB 
$41($ High $\times$ High $)$ and ADB $542 \times$ DB 16 (High $\times$ High) were identified as best specific cross combinations based on their sca effects and per se performance.

Standard heterosis over DCH 32 and Mahalakshmi ranged from -27.95 (TSH 0250 $\times$ TCB 37) to $30.09($ BS $37 \times$ SUVIN) and 38.84 (TSH $0250 \times$ TCB 37) to 10.43 (BS 37 $\times$ SUVIN), respectively. Forty one hybrids over DCH 32 and six hybrids over Mahalakshmi showed positive significant heterosis. The potential hybrids identified as per per se performance, sca effects and standard heterosis were, BS $37 \times$ SUVIN (79.98), H $1442 \times$ DB 16 (78.91) and BS $37 \times$ GSB 41(77.89). Similar results for number of bolls plant $^{-1}$ were earlier reported by Amalabalu et al., (2012) and Kumar et al., (2013) for heterosis over standard check.

\section{Boll weight (g)}

Estimates of $\mathrm{gca}$ effects in pooled analysis ranged from -0.33 (MCU 5) to 0.23 (MR 786) among lines and from -0.27 (SUVIN) to 0.20 (GSB 40) among testers. gca effects were positive and significant for the lines, MR 786 (0.23), ADB 532 (0.21), NDLH 1938 (0.14), ADB 542 (0.13), H 1442 (0.12) and BS 37 $(0.08)$ and in the testers GSB $40(0.20)$, DB $16(0.16)$ and DB 11 (0.03). The sca effects varied from -0.53 (L $762 \times$ GSB 41) to 0.86 (TSH $0250 \times$ GSB 40). Based on per se performance and sca effects the crosses viz., TSH $0250 \times$ GSB 40 (Low $\times$ High), H $1442 \times$ GSB $41($ High $\times$ Low) and ADB $542 \times$ DB 11 $($ High $\times$ High) were noticed as superior hybrids for boll weight.

Standard heterosis over DCH 32 and Mahalakshmi ranged from -23.36 (SCS $793 \times$ TCB 37) to 23.29 (TSH $0250 \times$ GSB 40) and -30.60 (SCS $793 \times$ TCB 37) to 11.63 (TSH $0250 \times$ GSB 40), respectively. Standard heterosis in desirable direction was observed in seventeen hybrids over DCH 32 and two hybrids over Mahalakshmi. Based on overall performance (per se performance, sca effects and standard heterosis) the best specific cross combinations identified were, TSH $0250 \times$ GSB 40 (4.21), H $1442 \times$ GSB 41(3.97) and ADB $532 \times$ GSB 40 (3.84). These results are in conformity with the reports of Kumar et al., (2013) and Nirania et al., (2014) over standard check.

\section{Lint index (g)}

Range of gca effects over locations for lint index was from -0.45 (BS 37) to 0.50 (L 1058) among lines and from -0.26 (SUVIN) to 0.38 (DB 16) among testers. Out of 12 lines, L 1058 (0.50), MR 786 (0.29), L 762 (0.24), ADB $532(0.15)$ and H $1442(0.10)$ recorded significant positive $g c a$ effects.

Whereas, in the testers DB $16(0.38)$, GSB 41 $(0.12)$ and GSB $40 \quad\left(0.06^{* *}\right)$ exhibited significant gca effects in desired direction for this character. The sca effects for lint index varied from $-0.65($ MR $786 \times$ DB 11) to 0.92 (TSH $0250 \times$ GSB 40). Estimated component of variance due to general combining ability (0.049) was lower than due to specific combing ability (0.058). Based on per se performance and $s c a$ effects, the crosses, TSH $0250 \times$ GSB $40($ Low $\times$ High $)$, L $762 \times$ SUVIN (High $\times$ Low) and WGCV $48 \times$ SUVIN (Low $\times$ Low) were detected as promising specific cross combinations.

Standard heterosis over DCH 32 was found in thirty two hybrids and over Mahalakshmi in one hybrid (L $1058 \times \mathrm{DB} 16)$. The best performing hybrids identified based on per se performance, sca effects and standard heterosis were ADB $532 \times$ DB 16 (6.02) and TSH $0250 \times$ GSB 40 (5.93). These results are in conformity with the reports of Amalabalu et al., (2012) and Kumar et al., (2013) for heterosis over standard check. 
Table.1 Analysis of variance of combining ability for different characters in inter-specific hybrids of cotton (G. hirsutum L. × G. barbadense L.) Over three locations (pooled) during kharif, 2014-15

\begin{tabular}{|c|c|c|c|c|c|c|c|c|c|c|c|}
\hline Source of variation & d.f & $\begin{array}{c}\text { Days to } \\
50 \% \\
\text { flowering }\end{array}$ & $\begin{array}{l}\text { Plant height } \\
\quad(\mathrm{cm})\end{array}$ & $\begin{array}{c}\text { No. of } \\
\text { monopodia } \\
\text { plant }^{-1}\end{array}$ & $\begin{array}{c}\text { No. of } \\
\text { sympodia } \\
\text { plant }^{-1}\end{array}$ & $\begin{array}{c}\text { No. of bolls } \\
\text { plant }^{-1}\end{array}$ & $\begin{array}{c}\text { Boll weight } \\
\text { (g) }\end{array}$ & $\begin{array}{c}\text { Lint index } \\
\text { (g) }\end{array}$ & $\begin{array}{c}\text { Seed index } \\
(\mathrm{g})\end{array}$ & $\begin{array}{c}\text { Ginning } \\
\text { out-turn } \\
(\%)\end{array}$ & $\begin{array}{c}\text { Seed cotton } \\
\text { yield plant }^{-1}(g)\end{array}$ \\
\hline Replications & 2 & 0.55 & 12.87 & 0.02 & 1.75 & 6.53 & 0.03 & 0.01 & 0.14 & 0.88 & 17.03 \\
\hline Environments & 2 & $989.97 * *$ & $27421.73 * *$ & $6.83 * *$ & $3763.31 * *$ & $13552.86^{* *}$ & $7.24 * *$ & $21.02 * *$ & $794.63 * *$ & $849.38 * *$ & $50063.40 * *$ \\
\hline Rep $\times$ Env & 4 & 1.86 & 87.39 & 0.00 & 1.23 & 18.03 & 0.01 & 0.02 & 0.12 & 0.78 & 52.96 \\
\hline Treatments & 89 & $120.90 * *$ & $3391.81 * *$ & $2.45^{* *}$ & $94.80 * *$ & $1316.79 * *$ & $1.48 * *$ & $2.23 * *$ & $13.73 * *$ & $47.86 * *$ & $13620.91 * *$ \\
\hline Parents & 17 & $397.79 * *$ & $1024.64 * *$ & $0.97 * *$ & $14.62 * *$ & $390.94 * *$ & $3.59 * *$ & $1.96 * *$ & $9.82 * *$ & $24.09 * *$ & $3930.51 * *$ \\
\hline Parents (Line) & 11 & $145.89 * *$ & $471.73 * *$ & $1.06^{* *}$ & $15.55^{* *}$ & $371.73 * *$ & $0.16^{* *}$ & $0.85^{* *}$ & $3.66^{* * *}$ & $12.17 * *$ & $2614.58 * *$ \\
\hline Parents(Testers) & 5 & $32.79 * *$ & $730.54 * *$ & $0.88 * *$ & $13.62 * *$ & $164.07 * *$ & $0.35 * *$ & $2.69 * *$ & $3.41 * *$ & $46.68 * *$ & $1471.83 * *$ \\
\hline Parents (L vs T) & 1 & $4993.78 * *$ & $8577.13^{* *}$ & $0.33 * *$ & $9.33^{*}$ & $1736.48 * *$ & $57.55^{* *}$ & $10.51 * *$ & $109.56 * *$ & $42.29 * *$ & $30699.13 * *$ \\
\hline Parent vs Crosses & 1 & $275.04 * *$ & $226538.63 * *$ & $87.55 * *$ & $5874.75 * *$ & $69282.50 * *$ & $4.12 * *$ & $25.12 * *$ & $720.54 * *$ & $777.65 * *$ & $451974.84 * *$ \\
\hline Crosses & 71 & $52.43^{* *}$ & $815.68 * *$ & $1.61 * *$ & $32.59 * *$ & $581.21 * *$ & $0.93 * *$ & $1.97 * *$ & $4.71 * *$ & $43.27 * *$ & $9767.15^{* *}$ \\
\hline Line effect & 11 & $110.73 * *$ & $1548.47 * *$ & $3.52 * *$ & $54.00 *$ & $1880.81 * *$ & $1.87 * *$ & $4.38 * *$ & $11.73 * *$ & $106.79 * *$ & $27448.06^{* *}$ \\
\hline Tester effect & 5 & 67.41 & $2364.03^{* *}$ & 0.56 & $74.91 *$ & 691.19 & $3.34 * *$ & $6.00 * *$ & 5.41 & $107.24 * *$ & $25580.27 * *$ \\
\hline Line $\times$ Tester effect & 55 & $39.41 * *$ & $528.37 * *$ & $1.32 * *$ & $24.45^{* *}$ & $311.29 * *$ & $0.53 * *$ & $1.12 * *$ & $3.25 * *$ & $24.75^{* *}$ & $4793.40 * *$ \\
\hline Env $\times$ Treatments & 178 & $17.55 * *$ & $618.07 * *$ & $1.21 * *$ & $22.67 * *$ & $372.60 * *$ & $0.30 * *$ & $0.57 * *$ & $4.64 * *$ & $11.37 * *$ & $3385.54 * *$ \\
\hline Env $\times$ Parents & 34 & $8.51 * *$ & 144.54 & $0.59 * *$ & $12.17 * *$ & $77.93 * *$ & $0.14 * *$ & $0.36^{* *}$ & $2.73 * *$ & $12.00 * *$ & $670.42 * *$ \\
\hline Env $\times$ Parents $(\mathrm{L})$ & 22 & 4.38 & 126.41 & $0.38 * *$ & $11.53 * *$ & $78.65 * *$ & $0.14 * *$ & $0.19 * *$ & $0.99 * *$ & $9.33 * *$ & $774.10 * *$ \\
\hline Env $\times$ Parents $(T)$ & 10 & 3.02 & $207.80 *$ & $0.67 * *$ & $12.40 * *$ & $85.92 * *$ & $0.14 * *$ & $0.71 * *$ & $4.05 * *$ & $17.34 * *$ & $512.51 * *$ \\
\hline $\operatorname{Env} \times \operatorname{PAR}(\mathrm{L} v s \mathrm{~T})$ & 2 & $81.33^{* *}$ & 27.69 & $2.40 * *$ & $18.18 * *$ & 30.10 & $0.06^{*}$ & $0.58 * *$ & $15.26^{* *}$ & $14.77 * *$ & 319.42 \\
\hline Env $\times$ Parent $v s$ Cross & 2 & $103.56^{* *}$ & $21608.40^{* *}$ & $12.05 * *$ & $620.45 * *$ & $4632.61 * *$ & $2.61 * *$ & $1.92 * *$ & $56.53 * *$ & $54.76^{* *}$ & $26339.47 * *$ \\
\hline Env $\times$ Crosses & 142 & $18.51 * *$ & $435.81 * *$ & $1.21 * *$ & $16.76^{* *}$ & $383.15^{* *}$ & $0.31 * *$ & $0.60 * *$ & $4.36^{* *}$ & $10.61 * *$ & $3712.35 * *$ \\
\hline Env $\times$ Line effect & 22 & $29.24 *$ & $1022.23 * *$ & $2.20 * *$ & $27.13 *$ & $749.16^{* *}$ & 0.29 & 0.65 & 3.55 & 12.89 & $6011.89 *$ \\
\hline Env $\times$ Tester effect & 10 & 18.04 & 127.42 & $1.90 *$ & 19.30 & 540.70 & 0.49 & 0.60 & 6.29 & 9.93 & 4450.07 \\
\hline Env $\times \mathrm{L} \times \mathrm{T}$ effect & 110 & $16.40 * * *$ & $346.56^{* *}$ & $0.95 * *$ & $14.45^{* *}$ & $295.63^{* *}$ & $0.29 * *$ & $0.59 * *$ & $4.35^{* *}$ & $10.21 * *$ & $3185.38 * *$ \\
\hline Error & 534 & 3.08 & 108.21 & 0.05 & 1.73 & 11.02 & 0.02 & 0.03 & 0.20 & 1.06 & 200.62 \\
\hline
\end{tabular}

* Significant at $5 \%$ level, **Significant at $1 \%$ level 
Table.2 General combining ability effects of parents for different characters in cotton over three locations (pooled) During kharif, 2014-15

\begin{tabular}{|c|c|c|c|c|c|c|c|c|c|c|c|c|}
\hline & & & $\begin{array}{c}\text { Days to } \\
50 \% \\
\text { flowering }\end{array}$ & $\begin{array}{l}\text { Plant height } \\
\text { (cm) }\end{array}$ & $\begin{array}{c}\text { No. of } \\
\text { monopodia } \\
\text { plant }^{-1}\end{array}$ & $\begin{array}{l}\text { No. of } \\
\text { sympodia } \\
\text { plant }^{-1}\end{array}$ & $\begin{array}{l}\text { No. of bolls } \\
\text { plant }^{-1}\end{array}$ & $\begin{array}{c}\text { Boll weight } \\
\text { (g) }\end{array}$ & $\begin{array}{c}\text { Lint index } \\
(\mathrm{g})\end{array}$ & $\begin{array}{l}\text { Seed index } \\
(\mathrm{g})\end{array}$ & $\begin{array}{l}\text { Ginning out- } \\
\text { turn }(\%)\end{array}$ & $\begin{array}{l}\text { Seed cotton } \\
\text { yield plant } \\
\text { (g) }\end{array}$ \\
\hline & \multicolumn{12}{|c|}{ LINES } \\
\hline & \multicolumn{2}{|l|}{ NDLH 1938} & $-0.64 *$ & $9.32 * *$ & $-0.31 * *$ & $0.40 *$ & $6.43 * *$ & $0.14 * *$ & -0.04 & $-0.49 * *$ & $0.65 * *$ & $25.23^{* *}$ \\
\hline & \multicolumn{2}{|l|}{ H 1442} & $-1.90 * *$ & -0.28 & $-0.22 * *$ & $0.91 * *$ & $6.54 * *$ & $0.12 * *$ & $0.10 * *$ & $-0.49 * *$ & $1.21 * *$ & $25.40 * *$ \\
\hline & \multicolumn{2}{|l|}{ ADB 542} & $0.96^{* *}$ & $3.32 *$ & $0.10^{* *}$ & 0.11 & $1.16^{*}$ & $0.13 * *$ & 0.03 & $-0.23 * *$ & $0.58 * *$ & $11.21 * *$ \\
\hline & \multicolumn{2}{|l|}{ WGCV 48} & 0.12 & $-7.63 * *$ & $0.36^{* *}$ & $-0.48 *$ & $-1.16^{*}$ & $-0.05 * *$ & 0.03 & 0.04 & 0.23 & $-7.19 * *$ \\
\hline & \multicolumn{2}{|l|}{ MR 786} & -0.28 & $7.35 * *$ & $-0.15^{* *}$ & $1.72 * *$ & $1.60 * *$ & $0.23 * *$ & $0.29 * *$ & $0.22 * *$ & $0.73 * *$ & $16.75 * *$ \\
\hline & \multicolumn{2}{|l|}{ TSH 0250} & -0.27 & 0.17 & $-0.24 * *$ & $-1.01 * *$ & $-4.43 * *$ & $-0.15^{* *}$ & $-0.18 * *$ & $-0.13 *$ & $-0.56^{* *}$ & $-20.20 * *$ \\
\hline & BS 37 & & $2.12 * *$ & -0.42 & $0.51 * *$ & 0.14 & $2.68 * *$ & $0.08 * *$ & $-0.45^{* *}$ & -0.01 & $-1.84 * *$ & $10.51^{* *}$ \\
\hline & SCS 793 & & -0.14 & -0.67 & 0.03 & $1.06 * *$ & $3.68 * *$ & $-0.30^{* *}$ & $-0.37 * *$ & $0.21 * *$ & $-1.73 * *$ & $-7.09 * *$ \\
\hline & MCU 5 & & $1.23 * *$ & $-8.56^{* *}$ & $-0.12 * *$ & $-1.91 * *$ & $-14.61 * *$ & $-0.33 * *$ & $-0.31 * *$ & $0.50 * *$ & $-2.15^{* *}$ & $-55.60 * *$ \\
\hline & L 762 & & $-2.88 * *$ & 1.89 & $-0.21 * *$ & -0.12 & $4.41 * *$ & $-0.07 * *$ & $0.24 * *$ & $-0.79 * *$ & $2.22 * *$ & $7.56^{* *}$ \\
\hline & SE (gi) & & 0.25 & 1.49 & 0.03 & 0.19 & 0.48 & 0.02 & 0.02 & 0.06 & 0.14 & 2.03 \\
\hline & & & & & & & TESTERS & & & & & \\
\hline & GSB 40 & & 0.25 & $5.96 * *$ & $0.07 * *$ & $0.33^{*}$ & $-1.05^{* *}$ & $0.20 * *$ & $0.06 * *$ & $0.28 * *$ & -0.16 & $7.58 * *$ \\
\hline & DB 16 & & $-1.09 * *$ & 1.84 & $-0.09 * *$ & $0.95 * *$ & $2.46 * *$ & $0.16^{* *}$ & $0.38 * *$ & 0.06 & $1.35^{* *}$ & $16.62 * *$ \\
\hline & DB 11 & & -0.34 & 2.00 & $-0.08 * *$ & -0.07 & $1.42 * *$ & $0.03 *$ & $-0.14 * *$ & -0.08 & $-0.38 * *$ & $4.83 * *$ \\
\hline & GSB 41 & & $-0.45^{*}$ & 1.50 & $0.04 *$ & $0.69 * *$ & $2.71 * *$ & 0.01 & $0.12 * *$ & $-0.38 * *$ & $1.08 * *$ & $8.90 * *$ \\
\hline & TCB 37 & & $1.15^{* *}$ & $-5.18 * *$ & -0.01 & $-1.24 * *$ & $-2.95 * *$ & $-0.13^{* *}$ & $-0.16^{* *}$ & $0.15^{* *}$ & $-0.84 * *$ & $-15.20 * *$ \\
\hline & SUVIN & & $0.48 * *$ & $-6.12 * *$ & $0.07 * *$ & $-0.66^{* *}$ & $-2.59 * *$ & $-0.27 * *$ & $-0.26^{* *}$ & -0.03 & $-1.05^{* *}$ & $-22.73 * *$ \\
\hline & SE (gj) & & 0.18 & 1.05 & 0.02 & 0.13 & 0.34 & 0.01 & 0.02 & 0.04 & 0.10 & 1.43 \\
\hline$\sigma^{2} g c a$ & & $0.523^{*}$ & 14.81 & -0.005 & $0.385^{*}$ & 7.7 & 0.02 & 0.049 & 0.0 & 1.00 & 242.90 & \\
\hline$\sigma^{2} s c a$ & & $2.557 * *$ & 20.20 & 0.041 & $1.111^{*}$ & 1.7 & 0.02 & 0.058 & -0.1 & 1.61 & 178.6 & \\
\hline
\end{tabular}

*Significant at $5 \%$ level, $* *$ Significant at $1 \%$ level 
Table.3 Specific combining ability effects of 72 inter-specific hybrids of cotton (G. hirsutum L. $\times$ G. barbadense L.) for days to $50 \%$ flowering, plant height, no. of monopodia plant ${ }^{-1}$, no. of sympodia plant ${ }^{-1}$, no. of bolls plant ${ }^{-1}$ and boll weight over three locations

(Pooled) during kharif, 2014-15

\begin{tabular}{|c|c|c|c|c|c|c|c|c|c|c|}
\hline & $\begin{array}{c}\text { Days to } \\
50 \% \\
\text { flowering }\end{array}$ & $\begin{array}{c}\text { Plant } \\
\text { height } \\
(\mathrm{cm})\end{array}$ & $\begin{array}{c}\text { No. of } \\
\text { monopodia }^{\text {plant }^{-1}}\end{array}$ & $\begin{array}{c}\text { No. of } \\
\text { sympodia } \\
\text { plant }^{-1}\end{array}$ & $\begin{array}{l}\text { No. of } \\
\text { bolls } \\
\text { plant }^{-1}\end{array}$ & $\begin{array}{c}\text { Boll } \\
\text { weight } \\
\text { (g) }\end{array}$ & $\begin{array}{c}\text { Lint } \\
\text { index }(g)\end{array}$ & $\begin{array}{c}\text { Seed } \\
\text { index }(g)\end{array}$ & $\begin{array}{c}\text { Ginning } \\
\text { out-turn } \\
(\%)\end{array}$ & $\begin{array}{l}\text { Seed } \\
\text { cotton } \\
\text { yield } \\
\text { plant }^{-1} \\
\text { (g) } \\
\end{array}$ \\
\hline $\begin{array}{l}\text { NDLH } 1938 \times \text { GSB } \\
40\end{array}$ & $1.43^{*}$ & $7.70 *$ & $0.15^{*}$ & -0.83 & 0.01 & 0.08 & -0.05 & $-0.44 * *$ & 0.28 & 6.26 \\
\hline NDLH $1938 \times$ DB 16 & 0.55 & -6.17 & $0.46^{* *}$ & -0.33 & -1.39 & 0.06 & $-0.34 * *$ & 0.19 & $-1.57 * *$ & 0.57 \\
\hline NDLH $1938 \times$ DB 11 & $-2.42 * *$ & $-20.14 * *$ & 0.07 & $-1.48 * *$ & -1.55 & $0.09 *$ & $0.30 * *$ & $-0.65 * *$ & $2.42 * *$ & -0.14 \\
\hline $\begin{array}{l}\text { NDLH } 1938 \times \text { GSB } \\
41\end{array}$ & 0.69 & $8.21 *$ & $-0.42 * *$ & $1.22 * *$ & 2.14 & 0.03 & $0.18^{* *}$ & 0.05 & 0.57 & 7.00 \\
\hline $\begin{array}{l}\text { NDLH } 1938 \times \text { TCB } \\
37\end{array}$ & $1.76^{* *}$ & $9.36^{*}$ & -0.07 & 0.34 & $4.90 * *$ & $-0.18 * *$ & $0.35^{* *}$ & 0.08 & $1.43 * *$ & 1.01 \\
\hline $\begin{array}{l}\text { NDLH } 1938 \times \\
\text { SUVIN }\end{array}$ & $-2.01 * *$ & 1.04 & $-0.18 *$ & $1.09 *$ & $-4.12 * *$ & -0.07 & $-0.43 * *$ & $0.77 * *$ & $-3.14 * *$ & $-14.71 * *$ \\
\hline H $1442 \times$ GSB 40 & $1.80 * *$ & $7.32 *$ & $0.35 * *$ & 0.06 & $2.61 *$ & $-0.26 * *$ & -0.02 & $0.62 * *$ & $-0.79 *$ & $-10.38 *$ \\
\hline H $1442 \times$ DB 16 & $-1.85 * *$ & $-19.10 * *$ & $-0.34 * *$ & $2.03 * *$ & $4.27 * *$ & $0.16^{* *}$ & $-0.27 * *$ & $-0.37 *$ & -0.51 & $25.28 * *$ \\
\hline H $1442 \times$ DB 11 & $1.84 * *$ & $16.57 * *$ & $0.35^{* *}$ & -0.90 & 1.66 & $-0.33 * *$ & $-0.14 *$ & -0.16 & -0.31 & $-16.70^{* *}$ \\
\hline H $1442 \times$ GSB 41 & $-5.72 * *$ & -0.54 & 0.08 & 0.15 & 0.30 & $0.54 * *$ & $0.37 * *$ & -0.16 & $1.67 * *$ & $34.97 * *$ \\
\hline H $1442 \times$ TCB 37 & 0.90 & 1.33 & 0.13 & -0.78 & $-6.72 * *$ & 0.02 & $0.13^{*}$ & -0.08 & 0.51 & $-21.01 * *$ \\
\hline H $1442 \times$ SUVIN & $3.02 * *$ & -5.57 & $-0.57 * *$ & -0.55 & -2.12 & $-0.10 *$ & -0.07 & 0.14 & -0.57 & $-12.15^{*}$ \\
\hline ADB $542 \times$ GSB 40 & 0.28 & -5.96 & $-0.78 * *$ & $-1.88 * *$ & $6.49 * *$ & $-0.33 * *$ & -0.06 & 0.22 & -0.59 & -0.50 \\
\hline ADB $542 \times$ DB 16 & $-1.93 * *$ & 6.52 & $0.26 * *$ & $3.55 * *$ & $7.25 * *$ & $0.11 *$ & $0.19 * *$ & $-0.88 * *$ & $2.13 * *$ & $28.56 * *$ \\
\hline ADB $542 \times$ DB 11 & -0.01 & 3.08 & $0.44 * *$ & -0.14 & 1.84 & $0.32 * *$ & $-0.18 * *$ & $0.42 * *$ & $-1.24 * *$ & $24.33 * *$ \\
\hline ADB $542 \times$ GSB 41 & $1.43^{*}$ & 0.18 & 0.02 & -0.14 & $-9.14 * *$ & $0.17 * *$ & $0.33 * *$ & $0.78^{* *}$ & -0.06 & $-19.14 * *$ \\
\hline ADB $542 \times$ TCB 37 & -0.73 & 4.81 & $0.18^{*}$ & 0.37 & $6.06 * *$ & $-0.14 * *$ & $-0.23 * *$ & -0.11 & $-0.80 *$ & 7.63 \\
\hline ADB $542 \times$ SUVIN & 0.95 & $-8.63 *$ & -0.12 & $-1.75^{* *}$ & $-12.50 * *$ & $-0.13 * *$ & -0.05 & $-0.41 * *$ & 0.56 & $-40.88 * *$ \\
\hline ADB $532 \times$ GSB 40 & $-2.31 * *$ & -0.51 & $-0.17 *$ & $1.79 * *$ & $4.86^{* *}$ & $0.13 * *$ & $0.33 * *$ & $0.53 * *$ & 0.42 & $23.70 * *$ \\
\hline ADB $532 \times$ DB 16 & -0.63 & $19.74 * *$ & $-0.67 * *$ & $1.81 * *$ & $-6.84 * *$ & $0.14 * *$ & $0.36 * *$ & $0.62 * *$ & 0.21 & $-15.22 * *$ \\
\hline ADB $532 \times$ DB 11 & -0.94 & 3.43 & 0.06 & 0.64 & 1.36 & -0.07 & $-0.39 * *$ & $-0.57 * *$ & $-0.71 *$ & 2.43 \\
\hline ADB $532 \times$ GSB 41 & 1.17 & -5.81 & $0.55 * *$ & $-2.76^{* *}$ & $-2.79 *$ & $-0.09 *$ & 0.04 & $-0.42 * *$ & $0.68 *$ & $-14.10 * *$ \\
\hline ADB $532 \times$ TCB 37 & $2.79 * *$ & $-11.39 * *$ & $0.21 * *$ & $-1.09 *$ & $2.89 *$ & $-0.16^{* *}$ & $-0.37 * *$ & -0.18 & $-0.95 * *$ & -0.69 \\
\hline ADB $532 \times$ SUVIN & -0.09 & -5.45 & 0.02 & -0.39 & 0.52 & 0.05 & 0.03 & 0.02 & 0.34 & 3.89 \\
\hline WGCV $48 \times$ GSB 40 & -0.77 & -1.38 & $-0.49 * *$ & $1.46^{* * *}$ & -0.73 & 0.05 & $-0.20 * *$ & 0.18 & $-0.99 * *$ & -3.85 \\
\hline WGCV $48 \times$ DB 16 & -1.10 & $9.13 *$ & $0.30 * *$ & 0.88 & 0.07 & -0.03 & $-0.28 * *$ & 0.03 & $-1.21 * *$ & -1.48 \\
\hline WGCV $48 \times$ DB 11 & -0.29 & -0.70 & 0.14 & $1.91 * *$ & $8.23 * *$ & -0.07 & $0.19 * *$ & $-0.54 * *$ & $1.77 * *$ & $20.56 * *$ \\
\hline WGCV $48 \times$ GSB 41 & $2.04 * *$ & -2.03 & -0.02 & $-2.91 * *$ & 0.70 & $-0.09 *$ & $-0.38 * *$ & $-0.37 *$ & $-0.95 * *$ & -4.52 \\
\hline
\end{tabular}




\begin{tabular}{|c|c|c|c|c|c|c|c|c|c|c|}
\hline WGCV $48 \times$ TCB 37 & 0.11 & -3.22 & $0.26 * * *$ & 0.38 & 0.52 & -0.01 & 0.07 & -0.05 & 0.14 & 1.42 \\
\hline WGCV $48 \times$ SUVIN & 0.01 & -1.78 & $-0.19 * *$ & $-1.74 * *$ & $-8.79 * *$ & $0.14 * *$ & $0.61 * *$ & $0.76^{* *}$ & $1.23 * *$ & $-12.12 *$ \\
\hline MR $786 \times$ GSB 40 & 0.41 & 5.19 & $-0.17 *$ & $-1.19 * *$ & $-4.56 * * *$ & -0.06 & -0.04 & 0.16 & -0.51 & $-19.65 * *$ \\
\hline MR $786 \times$ DB 16 & 0.09 & -4.15 & -0.04 & $-2.70 * *$ & $-2.46^{*}$ & -0.01 & 0.09 & 0.15 & 0.09 & -7.02 \\
\hline MR $786 \times$ DB 11 & 0.67 & -1.31 & 0.13 & -0.76 & -0.44 & $-0.12 * *$ & $-0.65 * *$ & 0.01 & $-2.77 * *$ & -6.70 \\
\hline MR $786 \times$ GSB 41 & $-2.10 * *$ & -2.39 & -0.11 & $5.60 * *$ & $2.73^{*}$ & $0.09 *$ & $0.18^{* *}$ & $-0.95 * *$ & $2.29 * *$ & $14.50 * *$ \\
\hline MR $786 \times$ TCB 37 & $-1.93 * *$ & 0.41 & -0.05 & 0.06 & $4.73 * *$ & -0.02 & $0.13^{*}$ & $1.18^{* * *}$ & $-1.23 * *$ & $10.26^{*}$ \\
\hline MR $786 \times$ SUVIN & $2.86^{* *}$ & 2.25 & $0.24 * *$ & $-1.00 *$ & 0.01 & $0.13 * *$ & $0.30 * *$ & $-0.55 * *$ & $2.13 * *$ & 8.60 \\
\hline
\end{tabular}

\begin{tabular}{|c|c|c|c|c|c|c|c|c|c|c|}
\hline & $\begin{array}{c}\text { Days to } \\
50 \% \\
\text { flowering }\end{array}$ & $\begin{array}{c}\text { Plant } \\
\text { height } \\
\text { (cm) }\end{array}$ & $\begin{array}{c}\text { No. of } \\
\text { monopodia }^{\text {plant }} \\
\text { pla }^{-1}\end{array}$ & $\begin{array}{c}\text { No. of } \\
\text { sympodia }^{\text {plant }} \\
\text { pla }^{-1}\end{array}$ & $\begin{array}{c}\text { No. of } \\
\text { bolls } \\
\text { plant }^{-1}\end{array}$ & $\begin{array}{c}\text { Boll } \\
\text { weight } \\
\text { (g) }\end{array}$ & $\begin{array}{c}\text { Lint } \\
\text { index } \\
(\mathrm{g})\end{array}$ & $\begin{array}{c}\text { Seed } \\
\text { index } \\
(\mathrm{g})\end{array}$ & $\begin{array}{c}\text { Ginning } \\
\text { out turn } \\
(\%)\end{array}$ & $\begin{array}{c}\text { Seed cotton } \\
\text { yield plant } \\
\text { (g) }\end{array}$ \\
\hline $\begin{array}{l}\text { TSH } 0250 \times \\
\text { GSB } 40\end{array}$ & $-4.27 * *$ & $10.79 * *$ & $0.74 * *$ & $1.94 * *$ & $9.14 * *$ & $0.86^{* *}$ & $0.92 * *$ & $\begin{array}{c}- \\
1.00 * *\end{array}$ & $5.17 * *$ & $76.56^{* *}$ \\
\hline $\begin{array}{l}\text { TSH } 0250 \times \text { DB } \\
16\end{array}$ & 0.40 & 3.84 & $0.46^{* *}$ & $-1.44 * *$ & -0.65 & $-0.25 * *$ & 0.06 & 0.09 & 0.26 & $-14.42 * *$ \\
\hline $\begin{array}{l}\text { TSH } 0250 \times \text { DB } \\
11\end{array}$ & $1.43 *$ & -2.66 & $-0.44 * *$ & $1.02 *$ & $3.58 * *$ & $-0.25 * *$ & $0.18 * *$ & $0.85 * *$ & -0.43 & -4.08 \\
\hline $\begin{array}{l}\text { TSH } 0250 \times \\
\text { GSB } 41\end{array}$ & $1.99 * *$ & -6.12 & -0.05 & -0.17 & $2.92 *$ & $-0.26 * *$ & $0.16 * *$ & -0.05 & -0.49 & -7.36 \\
\hline $\begin{array}{l}\text { TSH } 0250 \times \\
\text { TCB } 37\end{array}$ & $1.50 *$ & $-8.22 *$ & $-0.73 * *$ & -0.82 & $-13.96 * *$ & 0.01 & $0.39 * *$ & -0.20 & $-1.32 * *$ & $-41.37 * *$ \\
\hline $\begin{array}{l}\text { TSH } 0250 \times \\
\text { SUVIN }\end{array}$ & -1.05 & 2.37 & 0.02 & -0.53 & -1.02 & $-0.11^{*}$ & $0.62 * *$ & $0.31 *$ & $-3.20 * *$ & -9.33 \\
\hline BS $37 \times$ GSB 40 & $2.12 * *$ & -6.82 & $0.18 *$ & 0.29 & 0.58 & $0.12 * *$ & $0.44 * *$ & -0.29 & $2.56 * *$ & $11.66 *$ \\
\hline BS $37 \times$ DB 16 & -0.87 & 1.05 & -0.03 & -0.46 & $-4.56 * *$ & $-0.16^{* *}$ & -0.11 & 0.21 & $-0.78 *$ & $-22.53 * *$ \\
\hline BS $37 \times$ DB 11 & 0.38 & -1.99 & $-0.17 *$ & $-2.08 * *$ & $-10.99 * *$ & $0.24 * *$ & $\begin{array}{c}- \\
0.17 * *\end{array}$ & 0.05 & $-1.01 * *$ & $-23.42 * *$ \\
\hline BS $37 \times$ GSB 41 & $-1.51 *$ & 2.29 & -0.09 & $1.57 * *$ & $6.86^{* *}$ & -0.03 & $0.32 * *$ & $0.74 * *$ & 0.19 & $18.96 * *$ \\
\hline BS $37 \times$ TCB 37 & -0.11 & -1.67 & 0.04 & 0.58 & $-6.16^{* *}$ & -0.02 & $0.32 * *$ & $0.80 * *$ & $-2.56^{* * *}$ & $-13.30 * *$ \\
\hline BS $37 \times$ SUVIN & 0.01 & 7.14 & 0.07 & 0.11 & $14.26 * *$ & $-0.14 * *$ & $\begin{array}{c}- \\
0.16^{* *}\end{array}$ & $\begin{array}{c}- \\
1.52 * *\end{array}$ & $1.60 * *$ & $28.63 * *$ \\
\hline $\begin{array}{l}\text { SCS } 793 \times \text { GSB } \\
40\end{array}$ & $2.60 * *$ & -4.53 & $-0.23 * *$ & -0.17 & $-9.31 * *$ & $-0.15 * *$ & $0.47 * *$ & $0.56^{* * *}$ & -0.62 & $-30.63 * *$ \\
\hline $\begin{array}{l}\text { SCS } 793 \times \text { DB } \\
16\end{array}$ & $1.72 * *$ & 4.85 & $-0.33 * *$ & $-2.12 * *$ & 1.04 & $0.16^{* *}$ & $0.36^{* *}$ & 0.14 & $1.14 * *$ & $11.52 *$ \\
\hline $\begin{array}{l}\text { SCS } 793 \times \text { DB } \\
11\end{array}$ & $-1.25 *$ & 2.57 & $0.25 * *$ & -0.29 & $2.80 *$ & $0.18 * *$ & $0.50 * *$ & $0.55 * *$ & $1.10 * *$ & $16.08 * *$ \\
\hline
\end{tabular}


Int.J.Curr.Microbiol.App.Sci (2017) 6(8): 1722-1735

\begin{tabular}{|c|c|c|c|c|c|c|c|c|c|c|}
\hline $\begin{array}{l}\text { SCS } 793 \times \text { GSB } \\
41\end{array}$ & $-2.03 * *$ & 1.37 & -0.02 & $-1.13^{*}$ & $3.72 * *$ & $0.12 * *$ & $\begin{array}{c}- \\
0.36^{* *}\end{array}$ & $0.63 * *$ & $-2.30 * *$ & $16.64 * *$ \\
\hline $\begin{array}{l}\text { SCS } 793 \times \text { TCB } \\
37\end{array}$ & -1.19 & 0.65 & -0.04 & 0.49 & $-4.25 * *$ & $-0.26 * *$ & $0.33 * *$ & -0.24 & $1.62 * *$ & $-24.02 * *$ \\
\hline $\begin{array}{l}\text { SCS } 793 \times \\
\text { SUVIN }\end{array}$ & 0.15 & -4.91 & $0.36 * *$ & $3.22 * *$ & $6.00 * *$ & -0.05 & $\begin{array}{c}- \\
0.37 * *\end{array}$ & $\begin{array}{c}- \\
0.51 * *\end{array}$ & $-0.93 * *$ & $10.40 *$ \\
\hline $\begin{array}{l}\text { MCU } 5 \times \text { GSB } \\
40\end{array}$ & $1.56^{*}$ & -3.66 & $0.58 * *$ & 0.41 & $-3.67 * *$ & -0.06 & $\begin{array}{c}- \\
0.53 * *\end{array}$ & -0.19 & $-2.17 * *$ & $-17.65 * *$ \\
\hline MCU $5 \times$ DB 16 & -0.43 & 1.80 & 0.04 & 0.14 & 1.11 & $0.18 * *$ & $0.17 * *$ & $0.97 * *$ & $-0.81 *$ & 7.89 \\
\hline MCU $5 \times$ DB 11 & $1.60 *$ & -1.87 & $-0.53 * *$ & 0.56 & $-6.84 * *$ & $-0.17 * *$ & 0.09 & $\stackrel{-}{0.76^{* *}}$ & $1.64 * *$ & $-23.98 * *$ \\
\hline $\begin{array}{l}\text { MCU } 5 \times \text { GSB } \\
41\end{array}$ & $1.49 *$ & 4.97 & $-0.80 * *$ & $-1.18 *$ & -0.39 & -0.06 & 0.02 & -0.03 & 0.07 & -4.25 \\
\hline $\begin{array}{l}\text { MCU } 5 \times \text { TCB } \\
37\end{array}$ & $-3.11 * *$ & 0.15 & $0.59 * *$ & -0.19 & $5.27 * *$ & $0.16 * *$ & -0.04 & $0.55^{* * *}$ & $0.77 *$ & $23.91 * *$ \\
\hline $\begin{array}{l}\text { MCU } 5 \times \\
\text { SUVIN } \\
\end{array}$ & -1.10 & -1.39 & 0.13 & 0.25 & $4.52 * *$ & -0.05 & $0.29 * *$ & $0.56 * *$ & 0.50 & $14.08 * *$ \\
\hline $\begin{array}{l}\mathrm{L} 1058 \times \mathrm{GSB} \\
40\end{array}$ & $-2.42 * *$ & -4.28 & $0.17 *$ & $-1.88 * *$ & -1.46 & $-0.24 * *$ & 0.03 & $0.75 * *$ & $-1.18 * *$ & $-17.38 * *$ \\
\hline L $1058 \times$ DB 16 & $5.70 * *$ & $\begin{array}{c}- \\
11.79 * *\end{array}$ & 0.11 & -0.37 & -0.53 & $-0.24 * *$ & 0.07 & $\begin{array}{c}- \\
0.69 * *\end{array}$ & $1.40 * *$ & $-13.36 * *$ \\
\hline L $1058 \times$ DB 11 & $-1.83 * *$ & 1.68 & $-0.42 * *$ & 0.18 & -0.01 & $0.21 * *$ & 0.06 & 0.15 & -0.14 & $13.11 * *$ \\
\hline $\begin{array}{l}\text { L } 1058 \times \text { GSB } \\
41\end{array}$ & 0.06 & -3.56 & $0.38 * *$ & 0.47 & -1.24 & $0.11 *$ & $\begin{array}{c}- \\
0.36^{* *}\end{array}$ & -0.22 & $-1.07 * *$ & 0.53 \\
\hline $\begin{array}{l}\mathrm{L} 1058 \times \mathrm{TCB} \\
37\end{array}$ & 0.24 & $7.58 *$ & $-0.15^{*}$ & 0.45 & 0.74 & 0.06 & $0.20 * *$ & -0.01 & $0.67 *$ & 6.59 \\
\hline L $1058 \times$ SUVIN & $-1.75 * *$ & $10.37 * *$ & -0.09 & $1.14 *$ & $2.51 *$ & $0.10^{*}$ & 0.00 & 0.02 & 0.32 & $10.50 *$ \\
\hline $\mathrm{L} 762 \times \mathrm{GSB} 40$ & -0.44 & -3.85 & $-0.33 * *$ & 0.10 & $-3.96 * *$ & $-0.14 * *$ & $0.35^{* *}$ & 0.03 & $-1.58 * *$ & $-18.14 * *$ \\
\hline L $762 \times$ DB 16 & $-1.65 * *$ & -5.73 & $-0.21 * *$ & $-0.98 *$ & $2.69 *$ & $-0.12 * *$ & $\begin{array}{c}- \\
0.32 * *\end{array}$ & $\begin{array}{c}- \\
0.45 * *\end{array}$ & -0.36 & 0.22 \\
\hline L $762 \times$ DB 11 & 0.82 & 1.35 & 0.12 & $1.33 * *$ & 0.37 & -0.03 & $0.23 * *$ & $0.64 * *$ & -0.32 & -1.50 \\
\hline L $762 \times$ GSB 41 & $2.49 * *$ & 3.44 & $0.48 * *$ & -0.73 & $-5.80 * *$ & $-0.53 * *$ & $\begin{array}{c}- \\
0.17 * * \\
\end{array}$ & -0.01 & -0.58 & $-43.25 * *$ \\
\hline $\mathrm{L} 762 \times \mathrm{TCB} 37$ & -0.23 & 0.21 & $-0.36 * *$ & 0.21 & $5.96 * *$ & $0.57 * *$ & $0.14 *$ & $\begin{array}{c}- \\
0.64 * * \\
\end{array}$ & $1.71 * *$ & $49.58 * *$ \\
\hline L $762 \times$ SUVIN & -0.99 & 4.58 & $0.30 * *$ & 0.16 & 0.73 & $0.24 * *$ & $0.47 * *$ & $0.43 * *$ & $1.13 * *$ & $13.08 * *$ \\
\hline $\mathrm{SE}\left(\mathrm{s}_{\mathrm{ij}}\right)$ & 0.62 & 3.64 & 0.07 & 0.46 & 1.17 & 0.04 & 0.06 & 0.16 & 0.34 & 4.98 \\
\hline
\end{tabular}

*Significant at $5 \%$ level, **Significant at $1 \%$ level 
Table.4 The best heterotic inter-specific cotton hybrids identified for different characters based on overall performance over three Locations during kharif, 2014-15

\begin{tabular}{|c|c|c|c|c|c|}
\hline \multirow{2}{*}{ Character } & \multirow{2}{*}{ hybrids } & \multirow{2}{*}{ Per se performance } & \multirow{2}{*}{ SCA effect } & \multicolumn{2}{|c|}{ Standard heterosis } \\
\hline & & & & DCH 32 & Mahalakshmi \\
\hline \multirow{2}{*}{ Days to $50 \%$ flowering } & H $1442 \times$ GSB 41 & 55.67 & $-5.72 * *$ & $-7.22 * *$ & $-7.73 * *$ \\
\hline & $\mathrm{L} 762 \times \mathrm{DB} 16$ & 58.11 & $-1.65 * *$ & $-3.15^{*}$ & $-3.68 *$ \\
\hline \multirow{3}{*}{ Plant height $(\mathrm{cm})$} & NDLH $1938 \times$ GSB 40 & 194.54 & $7.70^{*}$ & $21.36 * *$ & $8.70 * *$ \\
\hline & NDLH $1938 \times$ GSB 41 & 190.59 & $8.21 *$ & $18.90 * *$ & $6.50 *$ \\
\hline & H $1442 \times$ DB 11 & 189.85 & $16.57 * *$ & $18.44 * *$ & $6.08 *$ \\
\hline \multirow{3}{*}{ No. of monopodia plant ${ }^{-1}$} & ADB $532 \times$ GSB 41 & 3.20 & $0.55^{*}$ & $87.94 * *$ & $16.86^{* *}$ \\
\hline & BS $37 \times$ GSB 40 & 3.18 & $0.18^{*}$ & $86.96 * *$ & $16.25 * *$ \\
\hline & WGCV $48 \times$ TCB 37 & 3.03 & $0.26 * *$ & $78.23 * *$ & $10.82 * *$ \\
\hline \multirow{3}{*}{ No. of sympodia plant ${ }^{-1}$} & MR $786 \times$ GSB 41 & 31.43 & $5.60 * *$ & $36.96 * *$ & $22.97 * *$ \\
\hline & ADB $542 \times$ DB 16 & 28.02 & $3.55 * *$ & $22.12 * *$ & $9.64 * *$ \\
\hline & H $1442 \times$ DB 16 & 27.29 & $2.03 * *$ & $18.96 * *$ & $6.81 * *$ \\
\hline \multirow{3}{*}{ No. of bolls plant ${ }^{-1}$} & BS $37 \times$ SUVIN & 79.98 & $14.26 * *$ & $30.09 * *$ & $10.43 * *$ \\
\hline & H $1442 \times$ DB 16 & 78.91 & $4.27 * *$ & $28.34 * *$ & $8.95 * *$ \\
\hline & BS $37 \times$ GSB 41 & 77.89 & $6.86 * *$ & $26.69 * *$ & $7.54 * *$ \\
\hline \multirow{3}{*}{ Boll weight (g) } & TSH $0250 \times$ GSB 40 & 4.21 & $0.86^{* *}$ & $23.29 * *$ & $11.63 * *$ \\
\hline & H $1442 \times$ GSB 41 & 3.97 & $0.54 * *$ & $16.14 * *$ & $5.15 * *$ \\
\hline & ADB $532 \times$ GSB 40 & 3.84 & $0.13 * *$ & $12.39 * *$ & 1.77 \\
\hline \multirow{2}{*}{ Lint index $(g)$} & ADB $532 \times$ DB 16 & 6.02 & $0.36 * *$ & $18.69 * *$ & 2.71 \\
\hline & TSH $0250 \times$ GSB 40 & 5.93 & $0.92 * *$ & $17.06^{* *}$ & 1.31 \\
\hline \multirow{3}{*}{ Seed index (g) } & ADB $532 \times$ GSB 40 & 14.34 & $0.53 * *$ & $24.59 * *$ & $3.73 *$ \\
\hline & ADB $532 \times$ DB 16 & 14.22 & $0.62 * *$ & $23.57 * *$ & 2.88 \\
\hline & MR $786 \times$ TCB 37 & 14.19. & $1.18 * *$ & $23.33 * *$ & 2.68 \\
\hline \multirow{3}{*}{ Ginning out-turn (\%) } & TSH $0250 \times$ GSB 40 & 33.49 & $5.17 * *$ & $9.43 * *$ & $11.92 * *$ \\
\hline & L $1058 \times$ DB 16 & 33.24 & $1.40 * *$ & $8.61 * *$ & $11.07 * *$ \\
\hline & MR $786 \times$ GSB 41 & 33.13. & $2.29 * *$ & $8.27 * *$ & $10.73 * *$ \\
\hline \multirow{3}{*}{ Seed cotton yield plant ${ }^{-1}(\mathrm{~g})$} & H $1442 \times$ GSB 41 & 253.36 & $34.97 * *$ & $45.61 * *$ & $27.18 * *$ \\
\hline & H $1442 \times$ DB 16 & 251.38 & $25.28 * *$ & $44.47 * *$ & $26.18 * *$ \\
\hline & TSH $0250 \times$ GSB 40 & 248.02 & $75.56^{* *}$ & $42.54 * *$ & $24.50 * *$ \\
\hline
\end{tabular}

*Significant at $5 \%$ level, **Significant at $1 \%$ level 


\section{Seed index (g)}

General combining ability effects for seed index in pooled analysis varied from -0.79 (L 762) to 0.89 (ADB 532) in lines and in testers it ranged from -0.38 (GSB 41) to 0.28 (GSB 40). The lines, ADB 532 (0.89), MCU 5 (0.50), L 1058 (0.28), MR $786(0.22)$ and SCS $793(0.21)$ and the testers GSB $40(0.28)$ and TCB $37(0.15)$ possessed significant positive gca effects for this character. The cross combination, BS $37 \times$ SUVIN (-1.52) recorded lowest sca effect whereas, the highest was exhibited by MR 786 $\times$ TCB 37 (1.18). The crosses, MR $786 \times$ TCB 37 (High $\times$ High), MCU $5 \times$ DB 16 (High $\times$ Low) and BS $37 \times$ TCB 37 (Low $\times$ High) were registered as good specific cross combinations as per their per se performance and sca effects.

The significant positive heterosis was detected in fifty nine hybrids over DCH 32 and it varied from -3.59 (BS $37 \times$ SUVIN) to 24.59 (ADB $532 \times$ GSB 40), one hybrid (ADB $532 \times$ GSB 40) over Mahalakshmi and it ranged from 19.73 (BS $37 \times$ SUVIN) to 3.73 (ADB $532 \times$ GSB 40). Based on per se performance, positive sca effects and standard heterosis superior cross combinations identified were, ADB $532 \times$ GSB 40 (14.34), ADB $532 \times$ DB 16 (14.22) and MR $786 \times$ TCB 37 (14.19). Similar type of results were earlier reported by Amalabalu et al., (2012), Kumar et al., (2013), Nirania et al., (2014) and Tuteja (2014) for heterosis over standard check.

\section{Ginning out-turn (\%)}

In pooled analysis $g c a$ effects for lines ranged from -2.15 (MCU 5) to L 762 (2.22) whereas, for testers it was from -1.05 (SUVIN) to 1.35 (DB 16). Significant positive $\mathrm{gca}$ effects was recorded by L 762 (2.22), L 1058 (1.45), H 1442 (1.21), MR 786 (0.73), NDLH $1938(0.65)$ and ADB $542(0.58)$ in lines and DB $16(1.35)$ and GSB 41 (1.08) in testers. Among the crosses sca effects varied from -3.20 (TSH 0250 $\times$ SUVIN) to $5.17($ TSH $0250 \times$ GSB 40). The superior specific cross combinations identified based on their per se performance and sca effects were, TSH $0250 \times$ GSB 40 (Low $\times$ Low), MR $786 \times$ GSB 41 (High $\times$ High) and ADB $542 \times$ DB $16($ High $\times$ High $)$.

The heterosis over DCH 32 and Mahalakshmi ranged from -22.21 (BS $37 \times$ TCB 37) to 9.43 (TSH $0250 \times$ GSB 40) and -20.45 (BS $37 \times$ TCB 37) to 11.92 (TSH $0250 \times$ GSB 40), respectively. Among 72 hybrids studied, nine hybrids over DCH 32 and twelve hybrids over Mahalakshmi exhibited significant positive heterosis. Best specific cross combinations detected based on overall performance for this trait were, TSH $0250 \times$ GSB 40 (33.49), L 1058 $\times$ DB 16 (33.24) and MR $786 \times$ GSB 41(33.13). These results are in conformity with the reports of Amalabalu et al., (2012) and Patel et al., (2012).

\section{Seed cotton yield Plant ${ }^{-1}(\mathrm{~g})$}

The gca effects among the lines ranged from 55.60 (MCU 5) to 25.40 (H 1442) and from 22.73 (SUVIN) to 16.62 (DB 16) in testers. Significant and positive gca effects were desirable for this trait and were noticed in the lines, H 1442 (25.40), NDLH 1938 (25.23), MR 786 (16.75), ADB 542 (11.21), BS 37 (10.51), L 762 (7.56) and ADB 532 (4.22) and the testers viz., DB 16 (16.62), GSB 41 (8.90), GSB 40 (7.58) and DB 11 (4.83). Among the cross combinations, sca effects varied from -43.25 (L $762 \times$ GSB 41) to 76.56 (TSH $0250 \times$ GSB 40). The crosses, H $1442 \times$ GSB 41 (High $\times$ High), TSH $0250 \times$ GSB 40 (Low $\times$ High) and ADB $542 \times$ DB 16 (High $\times$ High) were identified as best specific cross combinations as per their per se performance and sca effects.

Thirty four hybrids over DCH 32 and thirteen hybrids over Mahalakshmi showed significant positive heterosis. Based on per se performance, sca effects and standard heterosis superior specific cross combinations identified were, $\mathrm{H}$ $1442 \times$ GSB 41(253.36), H $1442 \times$ DB 16 (251.38) and TSH $0250 \times$ GSB 40 (248.02). Similar results for significant positive standard heterosis over check were reported by Nirania $e t$ al., (2014) and Tuteja (2014). 
The crosses between two low combiners producing crosses with significant $s c a$ effect revealed the role of non-additive inter-allelic interaction. This may be due to high nicking ability/genetic diversity of parents involved. This component may be exploited through heterosis breeding. The crosses between high $\times$ low or low $\times$ high combiners resulted in superior cross combinations due to complementary gene action which has arisen out of both additive and non-additive gene action. These crosses may likely to throw superior transgressive segregants for desirable traits. These components may be exploited by adopting breeding procedures like cyclic hybridization, biparental mating and diallel selective mating system. The cross combinations involving high $\times$ high general combiners producing crosses with significant sca effect revealed the role of additive and additive $x$ additive genetic component of variance which could be easily improved through simple selection procedures.

\section{Combining Ability Variances and Gene Action}

The estimate of $g c a$ and $s c a$ variances in pooled analysis is presented in table 4. General combining ability is generally associated with additive gene action, while specific combining ability is due to dominance and epistasis. In the present study sca variances were higher than gca variances in pooled analysis for days to $50 \%$ flowering, plant height, number of monopodia plant ${ }^{-1}$, number of sympodia plant ${ }^{-1}$, lint index, ginning out-turn, micronaire value, bundle strength, fibre elongation and lint yield plant $^{-1}$ indicating the predominance of nonadditive gene action for these characters and boll weight was controlled by both additive and non-additive gene actions whereas, additive gene action controls the remaining traits. Deosarkar et al., (2014), Yanal et al., (2013), Rajamani et al., (2014), Simon et al., (2013), Patel et al., (2014), Usharani et al., (2014), Patel et al., (2012), Baloch et al., (2014). The traits which are controlling by non-additive gene action may be exploited by biparental mating or diallel selective mating or heterosis breeding. For improvement of number bolls plant $^{-1}$, breeding programmes like population improvement as well as heterosis breeding can be employed for exploitation as it is under the control of both additive and non-additive gene actions.

Based on overall performance (per se performance, significant sca effects and standard heterosis) for seed cotton yield plant ${ }^{-1}$ in pooled analysis the hybrids, H $1442 \times$ GSB $41, \mathrm{H} 1442 \times$ DB 16 and TSH $0250 \times$ GSB 40 recorded high per se performance (253.36, 251.38 and 248.02), significant sca effects (34.97, 25.28 and 76.56) and standard heterosis over DCH 32 and Mahalakshmi $(45.61 \%$ \& $27.18 \%, 44.47 \%$ \& 26.18\%, $42.54 \%$ \& $24.50 \%$ ). Besides seed cotton yield plant ${ }^{-1}$ the hybrids exhibited good per se performance, significant sca effects and standard heterosis for other important yield contributing characters viz., number of sympodia plant ${ }^{-1}$, number of boll plant $^{-1}$, boll weight and ginning out-turn and fibre quality traits like $2.5 \%$ span length and micronaire value. The best heterotic cross combinations identified based on overall performance for different characters and these can be tested over seasons, location and years before their commercial cultivation are presented in table 4.

\section{References}

Amalabalu, P., Kavithamani, P.D., Ravikesavan, $\mathrm{R}$ and Rajarathinam, $\mathrm{S}$. 2012. Heterosis for seed cotton yield and its quantitative characters of Gossypium barbadense L. Journal of Cotton Research and Development. 26(1): 37-40.

Baloch, M.J., Solangi, J.A., Jatoi, W.A., Rind, I.H and Halo, F.M. 2014. Heterosis and specific combining ability estimates for assessing potential crosses to develop $F_{1}$ hybrids in upland cotton. Pakistan Journal of Agriculture, Agricultural Engineering and Veterinary Science. 30(1): 8-18.

Deosarkar, D.B., Deshmukh, J.D and 
Deshmukh, V.D. 2014. Combining ability analysis for yield and fibre quality traits in upland cotton (Gossypium hirsutum L.). Journal of Cotton Research and Development. 28(1): 18-23.

Kempthorne, O. 1957. An Introduction to Genetic Statistics. John Wiley and Sons Inc., New York. 458-471.

Kumar, S.K., Ashok Kumar, $\mathrm{K}$ and Ravikesavan, R. 2013. Genetic effects of combining ability studies for yield and fibre quality traits in diallel crosses of upland cotton (Gossypium hirsutum L.). African Journal of Biotechnology. 13(1):119-126.

Meredith, W.R. and Bridge, R. R. 1972. Heterosis and gene action in cotton $(G$. hirsutum L.). Crop Science. 12: 304- 310.

Nirania, K.S., Jain, P.P and Yadav, N.K. 2014. Genetic improvement for seed cotton yield and its component traits through heterosis breeding in Gossypium hirsutum L. Journal of Cotton Research and Development. 28(2): 220-222.

Patel, B.N., Patel, N.A., Soni, N.V and Dave, V.D. 2014. Genetic studies of quantitative traits in inter-specific hybrids of tetraploid cotton (Gossypium hirsutum L. $\times$ Gossypium barbadense L.). Electronic Journal of Plant Breeding. 5(4):708-715.

Patel, N.A., Patel, B.N., Bhatt, J.P and Patel, J.A. 2012. Heterosis and combining ability for seed cotton yield and component traits in inter-specific cotton hybrids (Gossypium hirsutum L. $\times$ Gossypium barbadense L.). Madras Agricultural Journal. 99(10-12): 649-656.

Rajamani, S., Gopinath, M and Reddy, K.H.P. 2014. Combining ability for seed cotton yield and fibre characters in upland cotton
(Gossypium hirsutum L.). Journal of Cotton Research and Development. 28(2): 207- 210.

Simon, S.Y., Kadams, A.M and Aliyu, B. 2013. Combining ability analysis in $\mathrm{F}_{1}$ hybrids of cotton (Gossypium hirsutum L.) by diallel method in Northern Nigeria. Greener Journal of Agricultural Sciences. 3(2): 90-96.

Singh, R.K and Chaudhary, B.D. 1977. Biometrical Methods in Quantitative Genetic Analysis. Kalyani Publishers, New Delhi. 215-218.

Singh, R.K and Chaudhary, B.D. 1985. Biometrical Methods in Quantitative Genetic Analysis. Kalyani Publishers, New Delhi. 205-214.

Sprague, G.F and Tatum, L.A. 1942. General vs Specific combining ability in single crosses of corn. Journal of American Society for Agronomy. 34: 923-932.

Tuteja, O.P. 2014. Studies on heterosis for yield and fibre quality traits in GMS hybrids of upland cotton (Gossypium hirsutum L.). Journal of Cotton Research and Development. 28(1): 1-6.

Usharani, K.S., Vindhyavarman, $\mathrm{P}$ and Amala Balu, P. 2014. Combining ability analysis in intra-specific $\mathrm{F}_{1}$ diallel cross of upland cotton (Gossypium hirsutum L.). Electronic Journal of Plant Breeding. 5(3): 467-474.

Yanal, A., Patil, S.S., Manjula, S.M., Patil, B.C., Nadaf, H.L., Nandihali, B.S. 2013. Development of heterotic groups $(G$. hirsutum vs $G$. barbadense) based on combining ability and inter-specific hybrids performance for yield and fiber quality traits. Molecular Plant Breeding. 4(24): 196-208.

\section{How to cite this article:}

Balakrishna, B., Chenga Reddy, K.V. Siva Reddy and Lal Ahamed, M. 2017. Genetic Studies for Yield and Yield Components in Inter-Specific Hybrids (G. hirsutum $\times$ G. barbadense) of Cotton Over Environments. Int.J.Curr.Microbiol.App.Sci. 6(8): 1722-1735. doi: https://doi.org/10.20546/ijcmas.2017.608.206 